(also known as "CKD”, "chronic renal disease", "CRD”, "chronic renal insufficiency", "chronic renal failure” and "chronic kidney failure"; includes "end-stage renal disease", also known as "end-stage kidney disease" and "kidney failure")

Note: Acute kidney disease and acute renal failure are addressed in the Acute Kidney Failure Fact Sheet and the Kidney Disease and Kidney Failure Advisory. Kidney transplantation is more fully addressed in the Organ Transplantation Fact Sheet.

Is the initiation of non-invasive dental hygiene procedures* contra-indicated? Possibly (e.g., immediately post-kidney transplantation, in which only emergency oral care is indicated)

- Is medical consult advised? Possibly (e.g., for kidney transplantation and immunosuppression considerations, or when medically unaddressed deteriorating kidney function is suspected).

Is the initiation of invasive dental hygiene procedures contra-indicated?** Possibly, depending on the degree of renal dysfunction and its management. Specifically, initiation is contra-indicated for patients/clients receiving dialysis or for whom kidney transplantation has occurred or is planned.

- Is medical consult advised?. Yes, for patients/clients receiving dialysis (e.g., about antibiotic prophylaxis and bleeding risk) or for those for whom kidney transplantation has occurred or is planned (e.g., about immunosuppression and infection considerations). It is also advised for patients/clients in whom the dental hygienist suspects severe or medically unaddressed deteriorating kidney function. In persons in whom CKD is an established diagnosis, consultation with the patient/client's nephrologist (kidney specialist) will shed light on co-morbidities, CKD sequelae, and the best timing of dental hygiene procedures.

- Is medical clearance required? Yes, for patients/clients receiving hemodialysis. The dental hygienist should confirm with the nephrologist that the patient/client is medically stable to receive dental hygiene (and dental) treatment, as well as ascertain if antibiotic prophylaxis is indicated. Hemoglobin and hematocrit levels, white blood cell count, platelet count, and coagulation tests may be indicated.

- Yes, for patients/clients receiving peritoneal dialysis. The dental hygienist should confirm with the nephrologist that the patient/client is medically stable to receive dental hygiene (and dental) treatment, as well as ascertain if antibiotic prophylaxis is indicated.

- Yes, for patients/clients who have undergone, or are about to undergo, kidney transplantation.

Corticosteroids (often prescribed for medical management of end-stage renal disease, as well as following kidney transplantation) may suppress adrenal function, predisposing the patient/client to adverse reaction (including potential circulatory collapse) from stress associated with emotional, physical (including infection), and surgical stress; thus, supplemental steroids may need to be administered.

- Is antibiotic prophylaxis required? ......................... Possibly, particularly if the patient/client has received a kidney transplant, or if the patient/client is receiving hemodialysis or peritoneal dialysis. In dialysis situations, antibiotic prophylaxis may be indicated to prevent either infective endocarditis or infection of the dialysis access ${ }^{1}$.

- Is postponing treatment advised? Possibly, depending on the degree of renal dysfunction and the form of management.

- For patients/clients undergoing hemodialysis, dental hygiene (and dental) treatment should be avoided after hemodialysis on the same day (especially within the first 6 hours afterward), because complications are associated with the use of anticoagulants (e.g., heparin) administered during dialysis therapy. Invasive procedures should be postponed until the day after dialysis. If a new dialysis access shunt is being placed in the patient/client, then dental hygiene treatment should be postponed two weeks. No dental/dental hygiene treatment should be performed if there is any infection or clotting at the site of the shunt.

1 In hemodialysis, access is vascular, typically via a surgically created arteriovenous fistula or graft placed in the forearm. In peritoneal dialysis, access is via a surgically placed abdominal catheter. Notably, on the basis of apparently low risk associated with oral bacteria, the American Heart Association's 2003 guidelines do not include a recommendation for prophylactic antibiotics before invasive dental procedures are performed on patients with intravascular access devices to prevent infective endocarditis or endarteritis except if an abscess is being drained. 


\section{CHRONIC KIDNEY DISEASE}

(also known as "CKD”, "chronic renal disease", "CRD”, "chronic renal insufficiency", "chronic renal failure" and "chronic kidney failure"; includes "end-stage renal disease", also known as "end-stage kidney disease" and "kidney failure")

\section{Is the initiation of invasive dental hygiene procedures contra-indicated?** (cont"d)}

- For patients/clients undergoing peritoneal dialysis, anticoagulation therapy is not required. However, peritonitis ${ }^{2}$ is a risk, and, if suspected, professional oral healthcare may have to be delayed.

- For patients/clients who have, or are about to receive, a kidney transplant professional oral healthcare should be individually tailored to the patient/client. As a general principle, elective dental hygiene (and dental) treatment should be avoided for 3 to 6 months post-transplant.

- With CKD graded stage 2 (i.e., kidney damage with normal or mild decrease in glomerular filtration rate [GFR], with GFR of $60-89 \mathrm{~mL} / \mathrm{min} / 1.73 \mathrm{~m}^{2}$ ) or better, problems do not generally arise in the provision of outpatient dental hygiene (and dental) care if the patient/client's disease is well controlled and conservative medical care is being provided. If the patient/client is in an advanced stage of kidney failure or has other comorbid conditions (e.g., diabetes, hypertension, or systemic lupus erythematosus), or if electrolyte imbalance is present, dental hygiene treatment may be best provided after physician consultation in a hospital-like setting. Deferral of treatment may be indicated until the systemic health status of the patient/ client has been ascertained and the CKD is adequately controlled.

\section{Oral management implications}

- The dental hygienist is potentially in a position to detect deteriorating kidney function, because worsening oral health may be an indicator of progression of CKD.

- The oral health status of patients/clients with advanced CKD (particularly those about to start dialysis) is often poor, and this necessitates oral hygiene instruction and frequent periodic recall appointments.

- For patients/clients with end-stage renal disease undergoing hemodialysis, dental hygiene appointments should ideally be scheduled on days following dialysis when the patient/client is rested and the anti-coagulant effects of heparin used in dialysis have dissipated.

- Kidney function is an important consideration for the dental hygienist (and dentist) because:

- dosages and dosing intervals of medications excreted by the kidneys (e.g., amoxicillin and cephalosporin antibiotics) should be correlated with kidney function ${ }^{3}$, and thus the dental hygienist should discourage CKD patients/clients from self-adjusting medications without input from the prescribing physician. Furthermore, tetracycline and aminoglycoside antibiotics should generally be avoided due to their nephrotoxicity.

- the kidney handles minerals such as calcium and phosphorus, and thus the dental hygienist should discourage CKD patients/clients from self-adjusting diet without input from a physician or registered dietitian with renal expertise.

- Commonly used over-the-counter pain-relievers (particularly non-steroidal anti-inflammatory drugs [NSAIDs] such as ibuprofen, naproxen, and aspirin) may worsen kidney function in patients/clients with existing CKD ${ }^{4}$, and the dental hygienist plays a role in risk reduction. The antiplatelet activity of aspirin is another reason to avoid its use in uremic ${ }^{5}$ patients/clients.

- Local anesthetic lidocaine is primarily eliminated/metabolized by the liver, rather than the kidney, and it is not removed by dialysis. In chronic renal disease, no local anesthetic dosage adjustment is typically required for most dental procedures when dose is kept to a safe minimum.

2 Peritonitis is a potentially fatal inflammation of the peritoneum, the thin layer of tissue that lines the inner wall of the abdomen and covers most of the abdominal organs. Usually caused by infection, signs/symptoms include abdominal pain, fever, nausea, vomiting, loss of appetite, and inability to pass stool. It occurs at a frequency of one case per peritoneal dialysis patient/client every 1.5 years.

3 Kidney function can be assessed via a variety of tests, including glomerular filtration rate (GFR), blood urea nitrogen (BUN), and serum creatinine.

4 Prolonged NSAID and/or acetaminophen use (particularly in drug combination formulations) may also contribute to the onset of CKD via analgesic nephropathy. While acetaminophen is generally the pain reliever of choice in patients/clients with CKD, it should be used carefully given its potential to worsen kidney function.

5 Uremia is the accumulation of nitrogenous waste materials in the blood, which alters the $\mathrm{pH}$ of saliva, as well as blood. 


\section{CHRONIC KIDNEY DISEASE}

(also known as "CKD", "chronic renal disease", "CRD”, "chronic renal insufficiency”, "chronic renal failure" and "chronic kidney failure"; includes "end-stage renal disease", also known as "end-stage kidney disease" and "kidney failure")

\section{Oral management implications (cont'd)}

- The dental hygienist should be aware that hemodialysis removes some drugs (not typically including local anesthetic agents) from the circulating blood, and this may shorten duration of effect of certain prescribed medications.

- While blood pressure should be measured before and during treatment, the dental hygienist should not take blood pressure readings in the dialysis access arm. (This could lead to occlusion or infection of the vascular access.)

- Xerostomia management advice to the patient/client may include sucking on ice chips to cope with uremia and strict fluid restriction. However, the dental hygienist should consult with the renal dietitian and nephrologist to ensure that all oral self-care recommendations and products are compatible with the patient/client's prescribed renal diet and medical treatment plan.

- While uremic stomatitis usually resolves after uremia is successfully treated, lesion healing can be assisted by dilute hydrogen peroxide gargles several times daily.

- Abnormal healing after extractions may occur.

- Panoramic radiographs can identify bone abnormalities.

\section{Oral manifestations}

- Up to $90 \%$ of patients/clients with renal insufficiency show oral signs and symptoms in soft and hard tissues, whether resultant from the disease itself, its co-morbidities, or from its treatment. While the oral hygiene of patients/clients receiving dialysis is often poor, hemodialysis reverses or ameliorates many of the severe oral pathologic changes associated with end-stage renal disease.

- Metallic taste results from increased concentration of urea in saliva and its subsequent breakdown to ammonium. Altered taste sensation to sweet and acid flavours may also occur.

- Halitosis can result from uremic fetor ${ }^{6}$.

- Red-orange discolouration of the oral mucosa and cheeks is associated with pruritus and deposition of carotene-like pigments resulting from decreased renal filtration.

- Xerostomia results from dietary fluid restrictions that reduce salivary flow. Thus, patients/clients with CKD have greater than normal deposits of dental calculus. Xerostomia may also result from side effects of medications (such as antihypertensive agents), salivary gland atrophy, and oral breathing secondary to lung perfusion problems.

- Uremic stomatitis may occur in severe renal failure. Red mucosa covered with gray exudates progresses to frank ulceration and sometimes bleeding. The lesions are very painful and usually appear on the ventral surface of the tongue and on anterior mucosal surfaces.

- "Uremic frost" may occasionally be seen on the oral mucosa in untreated uremia. Urea crystals appear as white patches on epithelial surfaces after evaporation of saliva.

- Enamel abnormalities include:

- enamel hypoplasia resulting from alterations in calcium and phosphorus metabolism when end-stage renal disease (ESRD) begins at an early age ${ }^{7}$; and

- erosions on the lingual surfaces of teeth due to acidic regurgitation, vomiting induced by uremia, and nausea associated with dialysis.

- Anemia, nearly universally present in all patients/clients with ESRD (i.e., those with less than $10 \%$ renal function), manifests as pallor of the oral mucosa, glossitis, and recurrent aphthous ulcers.

6 Uremic fetor is breath that smells like urine, which results from increased levels of urea in the saliva.

7 In the developing dentition, red-brown discolouration and slight delay in eruption may occur. 


\section{CHRONIC KIDNEY DISEASE}

(also known as "CKD”, "chronic renal disease", "CRD”, "chronic renal insufficiency”, "chronic renal failure" and "chronic kidney failure"; includes

"end-stage renal disease", also known as "end-stage kidney disease" and "kidney failure")

\section{Oral manifestations (cont'd)}

- Gingival bleeding, oral petechiae, and oral ecchymoses may occur due to platelet dysfunction and use of anticoagulants.

- Lichen planus ${ }^{8}$, black hairy tongue, geographic tongue, hairy leukoplakia, and pyogenic granulomas occur at increased frequency in patients/clients with CKD. As well, lichenoid eruptions manifesting as white patches are associated with the use of some antihypertensive medications (e.g., diuretics and beta-blockers).

- Other oral manifestations include periodontal disease, parotitis, and susceptibility to infections (including oral candidiasis and angular cheilitis, particularly in kidney-transplanted patients/clients on immunosuppressive therapy ${ }^{9}$ ), loss of bone in the jaw (particularly in the maxilla, with demineralization and cortical loss), pathologic tooth mobility and drifting, painful teeth, and premature tooth loss.

- Vitamin and mineral deficiencies (including B vitamins, vitamin C, iron, and zinc) are common in patients/clients on dialysis, which contribute to oral pathology. Various manifestations include angular cheilitis, filiform papillary atrophy (bald tongue), extraoral dermatitis, and peeling of the lips.

- Caries rates tend not to be elevated due to urea that inhibits bacterial growth and neutralizes bacterial plaque acids, as well as due to elevated salivary $\mathrm{pH}$. However, non-carious tooth tissue loss is more prevalent in patients/clients with CKD, and calculus formation is increased due to the presence of urea in saliva.

- Secondary hyperparathyroidism, a serious complication of CKD, manifests as areas of abnormal calcium leaching from osseous structures and as calcium deposits on and in teeth and soft tissues. In addition to dental calculus, calcium deposits may be visible on periapical radiographs as narrowing of the pulp chamber or abnormal calcifications in soft tissues, or as radiolucent cysts $^{10}$ in bones (particularly in the maxilla). Calcifications in the carotid arteries may be visible on panoramic radiographs. Other x-ray manifestations include loss of lamina dura, loss of trabecular pattern, and changes in bone density.

- Gingival hyperplasia may be caused by cyclosporine and other anti-rejection immunosuppressive medications used in kidney transplantation. It may also result from calcium channel blockers (e.g., nifedipine, amlodipine, verapamil, diltiazem, etc.) used to manage hypertension, and it is exacerbated by poor oral hygiene. The hyperplasia mainly affects the labial surface of the interdental papillae, although it may also extend to the gingival margins and lingual and palatal surfaces.

- Risk of oral cancer (and other malignancies) is elevated in transplanted patients/clients on immunosuppressive therapy.

\section{Related signs and symptoms}

- Chronic renal disease is the gradual loss of the ability of the kidneys' nephrons to remove wastes, concentrate urine, and conserve electrolytes. It is defined by some authorities as the presence of kidney damage or reduced kidney function for more than 3 months and requires either a measured or estimated glomerular filtration rate ${ }^{11}$ (eGFR) of less than $60 \mathrm{~mL} / \mathrm{min}$ per $1.73 \mathrm{~m}^{2}$, or the presence of abnormalities in urine sediment, renal imaging, or biopsy results.

- Between 3 and 4 million Canadians have some form of chronic kidney disease (including about 49,000 on dialysis or with a kidney transplant), and the number of Canadians being treated for renal failure has more than tripled over the past 25 years.

8 Lichen planus is a disease of unknown origin that affects the mucous membranes and/or skin. In the mouth, lichen planus can manifest as white, lacy patches; red, swollen tissues; or erosive or bullous lesions. The various oral manifestations often cause burning or pain.

9 Immunosuppressed patients/clients are also susceptible to cytomegalovirus (CMV) infections and herpes simplex virus (HSV) infections and reactivations.

10 also known as brown tumours or osteoclastomas

11 Degree of renal function is usually measured and monitored by glomerular filtration rate (GFR), which is an expression of the quality of glomerular filtrate created each minute in the kidney's nephrons. Chronic kidney failure is characterized by a gradual reduction in the number of functional nephrons. Stages of CKD range from Stage 1 with a GFR $\geq 90 \mathrm{~mL} / \mathrm{min}$ per $1.73 \mathrm{~m}^{2}$ (renal damage with a normal or increased GFR) to Stage 5 with a GFR $<15 \mathrm{~mL} / \mathrm{min}$ per $1.73 \mathrm{~m}^{2}$ (end-stage renal disease, in which a patient/client is unable to survive without dialysis; typically this equates to $<15 \%$ kidney function. 


\section{CHRONIC KIDNEY DISEASE}

(also known as "CKD”, "chronic renal disease", "CRD”, "chronic renal insufficiency”, "chronic renal failure" and "chronic kidney failure"; includes "end-stage renal disease", also known as "end-stage kidney disease" and "kidney failure")

\section{Related signs and symptoms (cont'd)}

CKD is much more common in adults than children, and just over half of newly diagnosed kidney failure patients/clients are aged 65 years or older. Many persons are not diagnosed until there is irreversible, bilateral damage to the kidneys. CKD occurs more frequently in boys than girls, but some studies suggest women are affected more than men. Risk factors include a family history of kidney disease and First Nations ancestry.

- While highly prevalent, CKD progresses to end-stage renal disease in only $3 \%$ of Canadian patients/clients. $90 \%$ of persons with CKD have relatively mild disease and are at low risk of progression to ESRD.

- The most common causes of CKD and progressive renal failure are diabetes mellitus and chronic hypertension, followed by glomerulonephritis ${ }^{12}$ and other glomerular diseases. Other causes include polycystic kidney disease, direct kidney trauma, medication toxicity, systemic lupus erythematosus, urinary tract obstruction, reflux nephropathy, and silica exposure.

- CKD usually develops slowly over many years in both kidneys, eventually manifesting with various signs/symptoms as kidney function deteriorates and uremia ${ }^{13}$ develops. In addition to weight loss, dizziness, cloudy or tea-coloured urine, and proteinuria $^{14}$, manifestations include:

- neurologic and muscular features, such as headaches, reduced mental concentration, drowsiness, fatigue, alteration of vision, sensory disturbances (peripheral neuropathy, often manifesting as cold sensation), muscle cramps, seizures, and coma;

- skeletal and soft tissue features, such as bone erosions and extraosseous calcifications;

- hematologic features, such as bleeding (due to platelet dysfunction ${ }^{15}$ and coagulopathy), anemia, leukopenia (decreased white blood cell count, sometimes involving lymphopenia), hematuria, and splenomegaly (enlarged spleen);

- dermatologic features, such as pruritus, bruising, pallor, hyperpigmentation, uremic frost ${ }^{16}$, and brown discolouration of the nails;

- metabolic features, such as thirst, nocturia (frequent urination at night), and polyuria or reduced urinary output;

- endocrinologic features, such as thyroid dysfunction, amenorrhea, and decreased libido;

- cardiovascular features, such as shortness of breath, chest pain related to pericarditis, and fluid retention (which manifests as swelling of legs, ankles, feet, hands, and/or face);

- pulmonary features, such as hyperventilation (i.e., Kusmaul's respiration, secondary to acidosis) and pulmonary edema;

- gastrointestinal features, such as anorexia, nausea, vomiting, diarrhea, and gastrointestinal bleeding; and

- immunologic features, such as tendency to infections.

- Coronary artery (which can manifest as angina and myocardial infarction, heart failure, cerebrovascular disease, and peripheral vascular disease are associated with CKD.

- Dialysis-related amyloidosis ${ }^{17}$ commonly occurs in persons on dialysis for more than 5 years. Deposition of proteins on joints and tendons causes pain and stiffness.

- In hemodialysis ${ }^{18}$, patients/clients are at increased risk of acquiring hepatitis $\mathrm{B}$, hepatitis $\mathrm{C}$, and human immunodeficiency virus (HIV) infection.

12 Glomerulonephritis is inflammation of the glomeruli (tiny filters) of the kidney.

13 Uremia is the resultant clinical syndrome from renal failure, encompassing retention of excretory products (waste and water) and interference with endocrine and metabolic functions.

14 Proteinuria (also known as albuminuria) is the presence of abnormal quantities of protein in the urine.

15 Hemodialysis causes the additional problem of platelet destruction due to the mechanical trauma of the procedure.

16 Uremic frost usually presents as adherent white patches on the skin, typically on the trunk and limbs. It is produced by residual urea crystals when perspiration evaporates.

17 Amyloidosis is a condition in which abnormal protein builds up in tissues and organs.

18 Hemodialysis cleanses the blood via its withdrawal from the patient/client by a machine (usually in a specialized clinic setting), passing it through a dialyzer ("artificial kidney") to remove waste and toxins, and then re-infusing it back into patient/client via a vascular access (often a surgically created fistula or shunt). Typically, this process is administered every 2 or 3 days for 3 to 4 hours. Most dialysis patients/clients in Canada receive hemodialysis, which is more effective than peritoneal dialysis. 


\section{CHRONIC KIDNEY DISEASE}

(also known as "CKD”, "chronic renal disease", "CRD”, "chronic renal insufficiency”, "chronic renal failure" and "chronic kidney failure"; includes "end-stage renal disease", also known as "end-stage kidney disease" and "kidney failure")

\section{Related signs and symptoms (cont'd)}

- In peritoneal dialysis ${ }^{19}$, patients/clients are at increased risk of developing an abdominal hernia.

- CKD progresses to end-stage renal disease in a minority of patients/clients. ESRD results from near-total or total permanent kidney failure, which leads to death unless hemodialysis, peritoneal dialysis, or kidney transplantation is undertaken.

- Patients/clients with ESRD can secondarily develop hypertension, diabetes, anemia ${ }^{20}$, hyperparathyroidism ${ }^{21}$, hyperthyroidism, and malnutrition.

- The average life expectancy of a patient/client on dialysis (without a kidney transplant) is about 5 years (ranging from 20 years if the person starts dialysis in his/her late twenties to 2 years if the person is aged older than 75 years). The most common causes of death among patients/clients with ESRD are cardiovascular disease (about 50\% of mortality) and infections.

\section{References and sources of more detailed information}

- College of Dental Hygienists of Ontario

https://www.cdho.org/Advisories/CDHO_Factsheet_Acute_Kidney_Failure.pdf https://www.cdho.org/Advisories/CDHO_Advisory_Kidney_Disease_and_Kidney_Failure.pdf

- Arora P, Vasa P, Brenner D, Iglar K, McFarlane P, Morrison H and Badawi A. Prevalence estimates of chronic kidney disease in Canada: results of a nationally representative survey. CMAJ. 2013 June;185(9):417-423. http://www.cmaj.ca/content/185/9/E417

- Baddour LM, Bettman MA, Bolger AF, et al. Nonvalvular cardiovascular device-related infections. Circulation. 2003;108 (16):2015-2031.https://www.ahajournals.org/doi/10.1161/01.CIR.0000093201.57771.47 (2003 Statement of the American Heart Association Science Advisory and Coordinating Committee)

- Budenz, AW. Local Anesthetics and Medically Complex Patients. Journal of the California Dental Association. 2000:Aug;28 (8):611-618. http://www.cda.org/Portals/0/journal/journal_082000.pdf

- Ziebolz D, Hraský V, Goralczyk A, Hornecker E, Obed A and Mausberg RF. Dental care and oral health in solid organ transplant recipients: a single center cross-sectional study and survey of German transplant centers. Transplant International (European Society for Organ Transplantation). 2011 Dec;24(2):1179-1188. http://onlinelibrary.wiley.com/doi/10.1111/j.1432-2277.2011.01325.x/full

- Proctor R, Kumar N, Stein A, Moles A and Porter S. Oral and Dental Aspects of Chronic Renal Failure. J Dent Res. 2005;84 (3):199-208. http://www.ugr.es/ jagil/chronic\%20renal\%20failure_JDR.pdf

- Cervero AJ, Bagan JV, Soriano YJ and Roda RP. Dental management in renal failure: Patients on dialysis. Med Oral Patol Oral Cir Bucal. 2008 July;13(7):E419-426. http://www.medicinaoral.com/medoralfree01/v13i7/medoralv13i7p419.pdf

19 Peritoneal dialysis (typically self-administered by the patient/client at home) cleanses the blood via filling the abdominal cavity with hypertonic dialysate solution, with the peritoneal lining acting as a membrane to filter waste and excess water from the blood into the dialysis fluid. The dialysis fluid is then drained from the surgically placed abdominal catheter and discarded. This form of dialysis is repeated 4 to 6 times every day in the more common continual ambulatory peritoneal dialysis (CAPD) without the use of a machine. In the older method of continuous cyclic peritoneal dialysis (CCPD), a peritoneal dialysis machine removes and replaces the dialysate solution during the evening/ night usually while the patient/client sleeps.

20 Diseased kidneys fail to make enough erythropoietin (EPO), which stimulates bone marrow to make red blood cells.

21 Also known as "renal osteodystrophy" and "mineral and bone disorder", secondary hyperparathyroidism in advanced CKD results from excessive secretion of parathyroid hormone (PTH). This occurs in response to decreased renal excretion of phosphorus in conjunction with decreased renal production of the active form of vitamin D, which has the effect of decreasing serum calcium. In an attempt to re-establish metabolic balance, increased PTH secretion occurs, causing hypercalcemia and weakened bones. Patients/clients with mineral and bone disorder must limit phosphorus intake. Renal dietitians affiliated with dialysis and transplant centres typically work with nephrologists and patients/clients to plan a kidney-friendly diet, which often restricts fluid intake, optimizes protein intake, and limits dietary phosphorus, sodium, and potassium. 
(also known as "CKD”, "chronic renal disease”, "CRD”, "chronic renal insufficiency", "chronic renal failure” and "chronic kidney failure”; includes "end-stage renal disease", also known as "end-stage kidney disease" and "kidney failure")

\section{References and sources of more detailed information (cont'd)}

- Akar H, Akar GC, Carrero JJ, Stenvinkel P and Lindholm B. Systemic Consequences of Poor Oral Health in Chronic Kidney Disease Patients. Clin J Am Soc Nephrol. 2011 Jan;6(1):218-226. http: //cjasn.asnjournals.org/content/6/1/218.full.pdf+html

- Piccoli GB, Alrukhaimi M, Liu ZH, Zakharova E, Levin A; World Kidney Day Steering Committee. What we do and do not know about women and kidney diseases; questions unanswered and answers unquestioned: reflection on World Kidney Day and International Woman's Day. BMC Nephrol. 2018;19(1):66. Published 2018 Mar 15. doi:10.1186/s12882-018-0864-y https: //www.ncbi.nlm.nih.gov/pmc/articles/PMC5856379/

- The Kidney Foundation of Canada

- Facing the Facts 2019 https://kidney.ca/CMSPages/GetFile.aspx?guid=e87e4b3b-1aa5-4c30-852c-356593451726

- Chronic Kidney Disease https://kidney.ca/CMSPages/GetFile.aspx?guid=9a5bb7de-9cf3-4859-ac39-a9f57429d900

- National Institute of Dental and Craniofacial Research (National Institutes of Health) https://www.nidcr.nih.gov/sites/default/files/2017-09/dental-management-organ-stem-cell-transplant.pdf https://www.nidcr.nih.gov/sites/default/files/2017-09/organ-stem-cell-transplant-mouth.pdf

- National Institute of Diabetes and Digestive and Kidney Diseases (National Institutes of Health) https://www.niddk.nih.gov/health-information/kidney-disease/chronic-kidney-disease-ckd

- UptoDate http://www.uptodate.com/contents/dialysis-or-kidney-transplantation-which-is-right-for-me-beyond-the-basics

- Mayo Clinic https: / /www.mayoclinic.org/tests-procedures/hemodialysis/about/pac-20384824 https://www.mayoclinic.org/tests-procedures/peritoneal-dialysis/about/pac-20384725

Medscape

https://emedicine.medscape.com/article/238798-overview\#showall (Chronic Kidney Disease) https://emedicine.medscape.com/article/984358-overview\#showall (Chronic Kidney Disease in Children) https://www.medscape.org/viewarticle/570239_10 (Preventive Healthcare in Chronic Kidney Disease and End-stage Renal Disease)

National Kidney Foundation https://www.kidney.org/sites/default/files/01-10-7278_HBG_CKD_Stages_Flyer3.pdf https://www.kidney.org/newsletter/women-kidney-disease

WebMD

https://www.webmd.com/cancer/lymphoma/amyloidosis-symptoms-causes-treatments\#1

Bowen DM (ed.) and Pieren JA (ed.). Darby and Walsh Dental Hygiene: Theory and Practice (5 $5^{\text {th }}$ edition). St. Louis: Elsevier Saunders; 2020.

Little JW, Falace DA, Miller CS and Rhodus NL. Dental Management of the Medically Compromised Patient ( $9^{\text {th }}$ edition). St. Louis: Elsevier Mosby; 2018.

Pickett FA and Gurenlian JR. Preventing Medical Emergencies: Use of the Medical History in Dental Practice ( $3^{\text {rd }}$ edition). Baltimore: Wolters Kluwer Health, 2015.

* Includes oral hygiene instruction, fitting a mouth guard, taking an impression, etc.

** Ontario Regulation 501/07 made under Dental Hygiene Act, 1991. Invasive dental hygiene procedures are scaling teeth and root planing, including curetting surrounding tissue.

Date: May 7, 2016

Revised: March 24, 2020; August 8, 2020 (limited update - primarily addition of references/links to Acute Kidney Failure Fact Sheet) 